\title{
Origen de los programas de cribado neonatal y sus inicios en España
}

\section{Origin of newborn screening programs and their beginnings in Spain}

doi.org/10.23938/ASSN.0012

E. Vicente ${ }^{1,2,3}$, L. Casas $^{2}$, E. Ardanaz ${ }^{1,3,4}$

\section{RESUMEN}

Los primeros programas de cribado neonatal de errores congénitos del metabolismo y otras enfermedades endocrinas comenzaron a finales de los años cincuenta y, una década más tarde, se introdujeron en España. Desde entonces han evolucionado mucho, pero de forma muy heterogénea, tanto a nivel internacional como autonómico. Los continuos avances científicos y tecnológicos, fundamentalmente desde el año 2000, han hecho que estas desigualdades se acentúen, haciendo de este tema un inagotable asunto de revisión, debate y discusión hasta nuestros días.

Esta revisión pretende recoger, de manera resumi$\mathrm{da}$, los orígenes de los programas de cribado neonatal y sus inicios en España, como un primer paso para el análisis de su desarrollo, evolución y situación actual, en unos años en el que se están implementando cambios en muchas comunidades españolas tras la publicación, en noviembre de 2014, de una orden estatal por la cual se regulan y tratan de homogeneizar.

\begin{abstract}
The first newborn screening programs to detect congenital errors of the metabolism and other endocrinal diseases started in the late 1950s and, one decade later, were introduced in Spain. Since then they have evolved considerably, but in a very heterogeneous way, at both the international level and the regional level. The continuous scientific and technological advances, basically since the year 2000 , have accentuated these inequalities, making this question into an inexhaustible subject of review, debate and argument up until the present.

This review aims to gather, in summarized fashion, the origins of the newborn screening programs and their beginnings in Spain as a first step in analyzing their development, evolution and current situation. This year updating is expected to take place in all the Spanish autonomous regions following publication in November 2014 of a state order that regulates and attempts to homogenize these programs.
\end{abstract}

1. Sección del Observatorio de la Salud Comunitaria. Servicio de Promoción de la Salud Comunitaria. Instituto de Salud Pública y Laboral de Navarra. Pamplona. Navarra.

2. Facultad de Ciencias de la Salud. Universidad Pública de Navarra. Pamplona. Navarra.

3. Instituto de Investigación Sanitaria de Navarra (IdiSNA). Pamplona. Navarra.

4. Centro de Investigación Biomédica en Red de Epidemiología y Salud Pública (CIBERESP).

\section{Correspondencia:}

Esther Vicente

Instituto de Salud Pública y Laboral de Navarra C/Leyre, 15

31003 Pamplona

E-mail: evicentc@navarra.es

Recepción: 03/02/2016

Aceptación provisional: 23/05/2016

Aceptación definitiva: 18/10/2016 


\section{INTRODUCCIÓN}

El 6 de noviembre de 2014 se publicó en el Boletín Oficial del Estado la Orden SSI/2065/2014, de 31 de octubre ${ }^{1}$, por la cual se regula el programa poblacional de cribado neonatal de enfermedades endocrinometabólicas, que forma parte de la cartera común básica de servicios asistenciales del Sistema Nacional de Salud, estableciendo la detección precoz de: hipotiroidismo congénito, fenilcetonuria, fibrosis quística, deficiencia de acil-coenzima A-deshidrogenasa de cadena media (MCADD), deficiencia de 3-hidroxi-acil-coenzima A-deshidrogenasa de cadena larga (LCHADD), acidemia glutárica tipo I (GA-I) y anemia falciforme. Asimismo, contempla la implantación de este programa de forma progresiva en todas las comunidades autónomas (CCAA) de manera que en el plazo de un año estén plenamente implementados sendos programas autonómicos.

Este trabajo pretende revisar algunos conceptos relacionados con este tema de gran actualidad como son: error congénito del metabolismo (ECM), screening y programa de cribado neonatal (PCN), para posteriormente repasar el origen de dichos PCN así como sus inicios en España, lo que dará pie a futuras revisiones que analicen la evolución a nivel autonómico de los mismos, la necesidad de regulación y el desarrollo de la implementación de la citada Orden SSI/2065/2014 en todo el país.

\section{Concepto de error congénito del metabolismo}

El término error congénito (o innato) del metabolismo fue introducido en 1908 por Archibald E. Garrod (Londres 1857-Cambridge 1936), en unas conferencias que impartió en el Royal College of Physicians, publicadas en 1909 en el libro Inborn Errors of Metabolism. Con él, hacía referencia a una serie de enfermedades infantiles que parecían ser debidas a una incapacidad innata para llevar a cabo algún proceso metabólico por ausencia o inactividad de una enzima y que se presentaban según un patrón de herencia mendeliano recesivo. Así describió algunos desórdenes metabólicos congénitos como alcaptonuria, albinismo, cistinuria o pentosuria ${ }^{2-4}$.

Este concepto ha evolucionado y actualmente el término ECM se utiliza para englobar aquellas enfermedades hereditarias que son consecuencia de alteraciones bioquímicas de origen génico en la estructura o función de una proteína. Son trastornos individualmente muy poco frecuentes pero colectivamente numerosos, ya que se han caracterizado más de 500 ECM distintos, pudiendo llegar a afectar a 1 de cada 500 recién nacidos. La mayoría de estas enfermedades son autosómico-recesivas, pero también hay algunas de carácter autosómico-dominante o de herencia ligada al cromosoma $\mathrm{X}^{5,6}$.

Una de las características más destacadas de los ECM es su gran heterogeneidad clínica debida tanto al tipo y función de la proteína alterada como a los distintos grados de afectación a nivel genético. A pesar de su heterogeneidad, muchos ECM presentan en común la posibilidad de tratamiento dietético o de sustitución enzimática y la gran carga sanitaria que suponen por su elevada frecuencia global y por las repercusiones a nivel del paciente, su familia y la sociedad en general ${ }^{6-8}$. Los continuos avances tecnológicos están permitiendo aumentar significativamente la identificación, detección, diagnóstico y tratamiento de los ECM, muchos de los cuales pueden producir consecuencias clínicas muy graves, o incluso la muerte, si no se interviene a tiempo ${ }^{6,9}$.

La baja prevalencia de cada uno de los ECM hace que queden englobados en las denominadas enfermedades raras ya que, según la definición más ampliamente adoptada en la Unión Europea, son aquellas alteraciones generalmente graves, crónicas y discapacitantes, que presentan menos de 5 casos por cada 10.000 habitantes ${ }^{10,11}$.

\section{Concepto de programa de cribado neonatal (PCN)}

En 1951, la Comisión de Enfermedades Crónicas de Estados Unidos definió screening como "la identificación presuntiva de 
enfermedades o defectos no reconocidos mediante pruebas, exámenes u otros procedimientos que pueden aplicarse rápidamente para distinguir a las personas en aparente buena salud que probablemente tengan una enfermedad de las que no la tengan. Una prueba de screening no está destinada a ser diagnóstica. Las personas con resultados positivos o sospechosos deben ser derivadas a sus médicos para el diagnóstico y tratamiento necesario" 12 .

En castellano se utilizan distintos términos para hacer referencia a este anglicismo, pero ninguno ha sido del todo generalizado o aceptado en su aplicación a la medicina; el más extendido es cribado, pero también se utilizan: pesquisa, selección, escrutinio, tría, triaje, tamiz, e incluso, tamizaje, a pesar de ser un vocablo inexistente"-13.

El cribado es una actividad de prevención secundaria de aplicación en enfermedades cuya prevención primaria no existe o es difícil de abordar. El principal objetivo del cribado es la detección precoz de un trastorno determinado en su período de latencia, asintomático o subclínico, con el fin de mejorar su pronóstico, disminuyendo la morbi-mortalidad asociada y/o aumentando la calidad de vida de las personas afectadas ${ }^{14-16}$. Pero el cribado no debe identificarse solo con la prueba en sí (de laboratorio, de imagen, el examen físico o un cuestionario), sino con un programa integral de carácter poblacional, como una actividad organizada y multidisciplinar cuya coordinación con el sistema sanitario asistencial resulta imprescindible ${ }^{17,18}$.

Antes de poner en marcha un programa de cribado se debe evaluar si cumple los criterios básicos para su implantación relativos a la enfermedad, la prueba y el propio programa; éstos pueden resumirse en: la enfermedad debe constituir un problema importante de salud (por su prevalencia y/o gravedad), con una historia natural bien definida y conocida, un largo período de latencia, criterios diagnósticos bien establecidos y tiene que ser fácilmente detectable por pruebas válidas, fiables, simples, seguras y aceptadas tanto por la población a la que van dirigidas como por el personal sanitario; además, debe haber un claro consenso respecto al proceso de confirmación, evidencia científica demostrada de que el tratamiento en fase pre-sintomática es más efectivo, así como una relación coste/efectividad y un balance riesgo/beneficio adecuados ${ }^{15,16,19-21}$.

En este contexto, los PCN se consideran una actividad esencial dentro de las actuaciones preventivas de salud pública. La Ley 14/2007, de 3 de julio, de investigación biomédica, los define como un tipo de "cribado genético", por lo que son objeto de las correspondientes consideraciones éticas y legales $^{22}$. El núcleo básico de estos PCN lo constituyen aquellas enfermedades endocrino-metabólicas en las que una detección y tratamiento precoces y adecuados evitan daños neurológicos, reducen la morbi-mortalidad y disminuyen las posibles discapacidades asociadas ${ }^{23}$.

Todo PCN debe garantizar el acceso equitativo y universal, con una cobertura del $100 \%$ de los recién nacidos en su ámbito geográfico y absoluta confidencialidad ${ }^{17}$.

Uno de los debates más actuales en la Administración Sanitaria tiene que ver con los dos tipos de PCN que se conocen: el PCN clásico, que sigue los criterios básicos ya mencionados, en el que para cada enfermedad cribada se necesita una muestra y un procedimiento de laboratorio; y el PCN ampliado, en el que con una única muestra y un único análisis, mediante espectrometría de masas en tándem, se pueden detectar decenas de ECM. Esto ha planteado una serie de problemas tanto desde el punto de vista ético como desde una perspectiva política, siendo el más importante la falta de homogeneidad en la aplicación de los PCN, ya que su contenido, práctica, protocolo y extensión varía en función del país y, más aún, de la comunidad autónoma en que se realice ${ }^{18}$.

\section{ORIGEN DE LOS PROGRAMAS DE CRIBADO NEONATAL}

Los orígenes de los PCN comenzaron con la detección precoz de la fenilcetonuria (PKU). En 1934, el químico noruego Ivar Asbjørn Følling identificó ácido fenilpirúvico (al que entonces llamaron "ácido idiota") en la 
orina de 2 hermanos de 6,5 y 4 años de edad, que presentaban un importante retraso mental, y cuya madre llevaba mucho tiempo diciendo e insistiendo a sus médicos que la orina de sus hijos olía raro, "como a moho". Para intentar confirmar la asociación entre retraso mental y presencia de ácido fenilpirúvico en la orina, Følling analizó 430 niños y niñas del área de Oslo con retraso mental e identificó 8 en cuya orina también se detectaba dicho ácido. Poco tiempo después publicó sus hallazgos, denominando imbecillitas phenylpyruvica a la enfermedad de estos niños y postulando que podía estar relacionada con un error en el metabolismo del aminoácido fenilalanina. Así fue la primera vez que se asoció la deficiencia mental con un ECM, el conocido como "oligofrenia fenilpirúvica", "enfermedad de Følling" o, más tarde, PKU (por la presencia en orina de fenilacetona $)^{24-26}$.

En 1951, el bioquímico inglés Louis I. Woolf sugirió en un artículo que el fallo intelectual de los fenilcetonúricos era debido a una intoxicación por ácido fenilacético y apuntaba a la reducción de fenilalanina en la ingesta como posible tratamiento: "Si la cantidad de fenilalanina y sus productos de degradación pueden reducirse, la función cerebral podrá resultar normal, el que esto lleve a la recuperación o no probablemente dependerá de la duración de la exposición del cerebro a la concentración dañina de la sustancia. Para alcanzar esta reducción, parecen merecedores de ser investigados dos posibles métodos: restringir la ingesta de fenilalanina a un mínimo básico en momentos precoces de la vida; e incrementar la velocidad de excreción de fenilalanina, administrando una sustancia que reduzca la reabsorción tubular competitivamente"27.

Unos meses más tarde, el médico alemán Hörst Bickel preparó un producto digerible y pobre en fenilalanina, a base de un hidrolizado de caseína tratado con carbón activo (que retiene los aminoácidos aromáticos), con el que trató a una niña de 2 años a la que había diagnosticado como fenilcetonúrica 8 meses antes. La paciente demostró una notable mejoría clínica en pocos meses, sufriendo una recaída cuando Bickel añadió fenilalanina a la dieta. Los resultados de este tratamiento dietético fueron publicados por Bickel y colaboradores en 1953 y $1954^{28-30}$.

En 1955, Woolf publicó un trabajo en el que hacía referencia al tratamiento dietético aplicado por Bickel, señalando la importancia de diagnosticar la PKU para pautar cuanto antes un tratamiento $\mathrm{y}$ evitar así las fatales consecuencias de la enfermedad. Expuso los aspectos económicos relacionados con la PKU y su tratamiento: por un lado, el bajo coste de los constituyentes de la dieta especial y, por otro, el importante ahorro en gastos de cuidados institucionales más la posible ganancia de miembros productivos para la sociedad. Así, recomendó lo que hoy se entiende por un programa de cribado ya que, decía, "el ensayo del fenilpirúvico en orina es extremadamente simple y las implicaciones de un resultado positivo son muy importantes" y además "si el coste del tratamiento está justificado, éste debe comenzar temprano". Propuso, también, realizar el análisis de orina de cada recién nacido a los 21 días de edad y, a los hermanos de fenilcetonúricos, hacerles un seguimiento diario desde su nacimiento para conocer a qué edad se eleva el nivel de fenilalanina en sangre y cuándo el ácido fenilpirúvico aparece por primera vez en la orina de los afectados ${ }^{31}$.

Con estas premisas, en marzo de 1958 comenzó en Cardiff el primer Programa de Detección de Fenilcetonuria con carácter oficial, pues implicaba a la Autoridad Sanitaria $^{32}$. Desde entonces se fueron implementando distintos programas de cribado en Reino Unido y Estados Unidos (EEUU), desarrollando distintas metodologías para el análisis de orina: gota de cloruro férrico al $10 \%$ sobre el pañal húmedo; tiras reactivas de papel impregnadas en cloruro férrico ("Phenistix") tanto para orina líquida como para pañal húmedo; muestra de papel impregnado en orina y dejado secar; empleo cromatografía en papel, etc. Fue Helen K. Berry (Cincinnati, EEUU), quien comenzó en 1958 a utilizar una muestra en fase sólida (orina secada en papel adsorbente) sobre la que se añadía una gota de cloruro férrico al $10 \%$. Además, cuando este ensayo 
resultaba dudoso o positivo, se examinaba la presencia de ácido o-hidroxifenilacético por cromatografía en papel de la muestra de orina impregnada en papel; este ácido es excretado en grandes cantidades en la orina de los fenilcetonúricos y es tan característico de la enfermedad como el ácido fenilpirúvico, aunque no se detecta tan fácilmente ${ }^{33}$. En base a estas experiencias se propuso la centralización de los ensayos para que fueran llevados a cabo por personas experimentadas, evitando falsos negativos.

En 1960, Willard R. Centerwall y col plantearon la ampliación del cribado a otras enfermedades basándose en la experiencia de Cincinnati, donde utilizaban papeles impregnados en orina para analizar, además, la presencia de proteínas y galactosa ${ }^{34}$.

En 1961 Woolf publicó una revisión de las técnicas existentes hasta ese momento para la detección de la PKU, haciendo énfasis en que con la muestra de orina seca impregnada en papel se podían analizar numerosas enfermedades, si bien entonces solo la consideraba adecuada para análisis de confirmación, ya que opinaba que en el Reino Unido no había laboratorios preparados para analizar las de todos los recién nacidos en el país. No fue hasta 1965 cuando se convenció del potencial de la muestra de orina en papel (conocida como "espécimen de Berry-Woolf") y de los laboratorios centralizados que realizaban la cromatografía en papel del ácido o-hidroxifenilacético, para superar los límites de detección del Phenistix y poder identificar "fenilcetonurias ocultas". Además, con este especimen, amplió las enfermedades que se podían detectar, añadiendo la glucosuria y cistinuria/homocistinuria a la proteinuria y galactosuria ya cribadas en el programa de Cincinnati ${ }^{26}$.

Mientras tanto, entre 1959 y 1961, el médico y microbiólogo americano Robert Guthrie desarrolló una prueba sencilla de inhibición bacteriana que permitía detectar la presencia de niveles elevados de fenilalanina en sangre neonatal utilizando papel de filtro como soporte de la muestra ${ }^{24}$. Guthrie, considerado impulsor de los PCN en EEUU, no quiso patentar su prueba, permitiendo así que estuviera disponible para todos los hospitales. El método de Guthrie es precisa- mente referenciado por Woolf en un capítulo del libro "Phenylketonuria" publicado en 1962, en el que comentaba la importancia de determinar la concentración de fenilalanina en sangre tanto en fenilcetonúricos tratados con una dieta baja en fenilalanina como en recién nacidos, ya que el ácido fenilpirúvico no aparece en orina hasta que la concentración en sangre de fenilalanina se ha elevado de forma considerable ${ }^{26}$.

El análisis de fenilalanina en sangre seca sobre papel absorbente ("espécimen de Guthrie") fue desplazando a las diversas pruebas de análisis de orina, lo que permitía llegar a todos los recién nacidos y centralizar los ensayos en un laboratorio. Posteriormente, gracias a la modificación realizada por Mary L. Efron en 1964, esta misma muestra se utilizó para la detección de diversos trastornos del metabolismo de los aminoácidos por cromatografía en papel ${ }^{26}$.

En 1961 Bickel introdujo el método de Guthrie en Europa. Ayudado por el propio Guthrie, montó en Marburg (Alemania) el primer laboratorio europeo de cribado neonatal que comenzó a recibir muestras en 1962. España fue uno de los pocos países europeos en los que la influencia de Guthrie apenas se notó ${ }^{24}$.

En 1968 se publicó una revisión de Woolf titulada Mass Screening of the Newborn of Metabolic Disease, en la que hacía referencia tanto a la muestra de orina como a la de sangre obtenida por punción del talón del recién nacido, impregnadas en papel y analizadas por los diversos métodos aplicables a cada muestra. Además, opinaba lo siguiente: "la fenilcetonuria es solamente uno entre una serie de errores innatos del metabolismo, que pueden tratarse con una dieta apropiada desde la temprana infancia, previniendo consecuencias clínicas horribles. Es un accidente de la historia que la fenilcetonuria haya recibido mucha atención y otras condiciones hayan sido largamente olvidadas. Es posible con poco coste adicional, ensayar para otras condiciones, los especímenes de sangre u orina recogidos para el cribado de fenilcetonuria. Varios métodos cromatográficos son particularmente apropiados para esto". Según Woolf, mediante el ensayo de orina, además de la PKU, se deberían de- 
tectar: tirosinosis, histidinemia, homocistinuria, cistinuria, galactosemia y disfunción tubular renal; y, utilizando la técnica de cromatografía de sangre, se podrían identificar: leucinosis, homocistinuria, hiperglicinemia, hiperprolinemia, hidroxiprolinemia y, con algo menos de fiabilidad, tirosinosis e histidinemia $^{35}$. Desde entonces, tanto el espécimen de Berry-Woolf como el espécimen de Guthrie se utilizaron para la determinación de multitud de magnitudes biológicas a través de numerosos métodos analíticos.

La ampliación más importante del cribado neonatal se produjo en Québec en 1973, cuando el médico endocrinólogo canadiense Jean H. Dussault adaptó un método de radioinmunoensayo para determinar el descenso de tiroxina (T4) en el espécimen Guthrie logrando la detección neonatal del hipotiroidismo congénito ${ }^{36}$. El hipotiroidismo congénito cumple, incluso mejor que la PKU, con todos los requisitos exigidos a una enfermedad para ser incluida en un PCN. El valor del punto de corte de T4 no se diferenciaba demasiado del límite inferior del intervalo de referencia por lo que en Europa y Japón se prefirió la determinación del incremento de tirotropina en sangre porque discriminaba con mejor sensibilidad diagnóstica ${ }^{24}$.

\section{INTRODUCCIÓN DE LOS PROGRAMAS DE CRIBADO NEONATAL EN ESPAÑA}

El introductor de los PCN en España fue el profesor Federico Mayor Zaragoza quien, en 1968, siendo catedrático de Bioquímica y rector de la Universidad de Granada, estableció el primer PCN español en dicha ciudad utilizando como muestra el espécimen de Berry-Woolf ${ }^{24}$.

En el curso académico 1966-67, Mayor Zaragoza realizó una estancia en Oxford con el Prof. Hans Krebs y allí coincidió con Louis I. Woolf, quien le hizo partícipe del "Programa de Tría Urinaria de Metabolopatías" que había puesto a punto en 1965 y que incluía la detección de PKU, tirosiluria, histidinemia, galactosemia, glucosuria, cistinuria/homocistinuria y proteinuria en muestras de orina recogidas sobre papel cromatográfico. Cuando volvió a España, Mayor Zaragoza expuso la iniciativa de Woolf en la entonces Dirección General de Sanidad y propuso un Plan para la investigación y control de las alteraciones congénitas metabólicas y cromosómicas, consiguiendo poco después implantar el mencionado PCN para el que puso en marcha, en 1969, el Centro de Investigación de Alteraciones Moleculares (CIAMYC) ${ }^{24,26}$.

Paralelamente en Barcelona, Juan Sabater Tobella pronunció una conferencia sobre ECM y retraso mental en 1968, siendo entonces desconocedor de la iniciativa del profesor Mayor Zaragoza en Granada. Entre los asistentes a la conferencia se encontraba Jesús Raventós, hombre muy influyente que había creado con sus propios recursos el primer centro de atención a deficientes mentales en Cataluña y que quedó encantado con la idea del cribado neonatal. Raventós consiguió acordar con la Fundación Juan March y la Diputación de Barcelona la dotación de un centro dedicado a este tema en la Maternidad Provincial de Barcelona, contratando a Sabater para poner a punto las técnicas de cribado de, entre otras, PKU y galactosemia, y analizar a los recién nacidos de la maternidad. Entonces, Sabater realizó una estancia de varios meses en el Centro de Metabolopatías del Children's Hospital de Boston, donde conoció a Mary Efron y Vivian Shih; y visitó a Charles Scriver en el Laboratorio del Children's Hospital de Montreal, responsable del programa de detección precoz del estado de Quebec. Con estas experiencias, Sabater presentó un proyecto a la Diputación de Barcelona y a la Fundación Juan March, las cuales financiaron la construcción de un edificio para el laboratorio y el coste de toda la instrumentación, respectivamente. Así, a mediados de 1969, empezó a funcionar el Instituto Provincial de Bioquímica Clínica. Desde un principio se realizó la cromatografía de aminoácidos en papel con la muestra de orina (recogida a los 29 días de vida) y se utilizó, hasta 1974, el test de Guthrie para la detección de PKU. A partir de entonces se analizaron simultánea- 
mente muestras de sangre y de orina por cromatografía en papel ya que los métodos microbiológicos se consideraron más engorrosos y menos informativos ${ }^{24,26}$.

En 1973, Mayor Zaragoza se trasladó a la Universidad Autónoma de Madrid y, con él, varios de sus discípulos de Granada, quienes sentían el compromiso de extender el programa de prevención de enfermedades metabólicas a toda España, empezando de forma inmediata por Madrid. Entre dichos colaboradores se encontraba Magdalena Ugarte Pérez quien, influenciada por su experiencia en técnicas cromatográficas aplicadas a muestras de orina en papel, fue la responsable de la introducción del espécimen de Guthrie en Granada para su análisis mediante cromatografía planar en capa fina. Esta metodología fue la que trasladó a Madrid y la que se empleó posteriormente de forma mayoritaria en toda España ${ }^{24,26}$.

A partir de estos primeros focos fueron surgiendo otros laboratorios en España, como el Centro de Bioquímica y Genética Clínica creado en 1975 por la Diputación Provincial de Murcia bajo la dirección de José Antonio Lozano Teruel.

Ya en el año 1974, el entonces Real Patronato de Educación Especial junto con otras entidades de carácter privado como la Fundación General Mediterránea, comenzaron a interesarse por el cribado neonatal. La prevención de la subnormalidad era un tema apenas conocido en aquellos años, pero tomó tal trascendencia que hizo que se desencadenasen una serie de hechos importantes ${ }^{24}$.

\section{PLAN NACIONAL DE PREVENCIÓN DE LA SUBNORMALIDAD (PNPS)}

En 1976, el Real Patronato de Educación Especial (ahora conocido como Real Patronato sobre Discapacidad) encargó a Mayor Zaragoza, miembro del Comité Ejecutivo, la creación de un grupo de trabajo para la confección y elaboración de un Plan Nacional de Prevención de la Subnormalidad (PNPS). Este grupo contaba con representantes de los organismos estatales competentes en materia de prevención, así como del Consejo Superior de Investigaciones Científicas (CSIC), de varias sociedades científicas médicas, de universidades, de hospitales y de centros asistenciales que, según aspectos científicos y de competencia profesional, se distribuyeron en tres ponencias: metabólico-genética (de la que fue secretario Juan Sabater), nutricionalpediátrica y perinatológica. Cada una de ellas tuvo que examinar y proponer posibles medidas y acciones de su campo específico, las cuales fueron recogidas en su conjunto en el borrador del PNPS que quedó finalmente aprobado en $1977^{24}$ y para el cual se creó una tasa de financiación de sus acciones por el Real Decreto 1762/1977 37 .

El PNPS centró su campo de actuación en la prevención de la subnormalidad, siendo sus líneas más importantes la mejora de la asistencia médica al embarazo, el parto y el puerperio, y la detección precoz neonatal de ECM. En lo concerniente a esta última, el PNPS se propuso como objetivos: potenciar y coordinar los centros de diagnóstico, selección y orientación precoz ya existentes y determinar áreas geográficas de actuación, crear nuevos centros por áreas y elaborar un directorio informativo. Así, se preveía la existencia de 11 laboratorios que, exceptuando el de las Islas Canarias y dada la natalidad de entonces, analizarían unos 50.000 recién nacidos al año ${ }^{24}$. También se plantearon una serie de objetivos fundamentales para el buen funcionamiento del programa de detección neonatal, que se podían resumir en 3 puntos y que hoy en día siguen siendo totalmente válidos ${ }^{38,39}$ :

- La detección precoz neonatal debe dar cobertura al $100 \%$ de los recién nacidos vivos en el área geográfica de influencia de los centros de detección neonatal.

- Se debe asegurar el tratamiento y seguimiento clínico de todos los recién nacidos detectados como positivos para cualquiera de las alteraciones analizadas.

- El tratamiento de los casos positivos detectados debe iniciarse lo antes posible y siempre antes del primer mes de vida. 
En 1976, el grupo de Gabriella Morreale de Castro y su marido Francisco Escobar del Rey, de la Facultad de Medicina de la Universidad Autónoma de Madrid, inició un estudio piloto de detección precoz de hipotiroidismo congénito en recién nacidos basado en la prueba del talón, con vistas a prevenir una deficiencia psíquica profunda mediante el tratamiento inmediato con hormona tiroidea. Gracias al importante esfuerzo del grupo de Morreale y de la ayuda del Real Patronato de Educación y Atención a Deficientes, el estudio se fue extendiendo progresivamente y se pudo implementar este Programa de Detección Precoz de Hipotiroidismo Congénito a nivel nacional. Dicho Programa se instauró en España antes que en otros países europeos y americanos, razón por la que se formó parte de los primeros Comités Internacionales para la organización de reuniones y conferencias sobre detección neonatal tiroidea ${ }^{26,40}$. Pocos años después UNICEF adoptó la prueba y comenzó a aplicarla en todo el mundo ${ }^{41}$.

Los estudios y trabajos del grupo de Morreale sobre la relación entre hipotiroidismo y deficiencia de yodo respaldaron la utilización de sal yodada por parte de la población española como medida de prevención del hipotiroidismo. Además, Morreale demostró el papel fundamental que la hormona tiroidea materna ejerce en el desarrollo del cerebro del feto ${ }^{40}$, lo que propició la suplementación con yodo a todas las embarazadas de forma sistemática. Gracias a sus investigaciones, desde 1990, la Organización Mundial de la Salud recoge en su tabla de derechos el consumo de yodo durante el embarazo y la primera infancia ${ }^{42}$.

Paralelamente, en 1977, se puso en marcha el laboratorio de Valencia y, un año después, por iniciativa de José Peña Guitián, José $M^{a}$ Fraga Bermúndez y José Ramón Alonso Fernández, el de Santiago de Compostela, contando con el asesoramiento de $\mathrm{M}^{\mathrm{a}}$ José García Muñoz y Antonio Maya Victoria, responsables directos de los centros de Madrid y Barcelona, respectivamente, y que también orientaron a otros muchos ${ }^{26}$.

Precisamente en 1978 se publicó el Real Decreto 2176/1978 por el que se encomendaba la realización y gestión del PNPS al
Ministerio de Sanidad y Seguridad Social ${ }^{43}$. Una de las acciones que regulaba era el establecimiento de una red técnica que permitiera la detección precoz de alteraciones metabólicas en el recién nacido. La red se desarrolló a buen ritmo, pasando de tener cinco centros de análisis que cubrían al 2,45 \% de los recién nacidos en España en 1979, a duplicar el número de centros y alcanzar una cobertura del $25 \%$ de la población neonatal al año siguiente. En 1981 la cobertura podía cifrarse en cerca del $31 \%$; y en 1982 eran 12 los centros donde se realizaba tanto el diagnóstico precoz de la PKU como el del hipotiroidismo congénito, aunque el territorio que abarcaba cada centro de cribado no se correspondía con el delimitado por cada una de las actuales $\mathrm{CCAA}^{24}$.

Mientras tanto, en 1980, se constituyó el Consejo Nacional de Prevención de la Subnormalidad, como órgano asesor del Real Patronato, para el impulso y orientación de la aplicación y ejecución del Plan, lo que vino haciéndose hasta 1982. Este Consejo fue presidido por José Zamarriego, ilustre obstetra, con amplísima participación de profesionales clínicos e investigadores bioquímicos que abarcaban todo el espectro de competencias. Precisamente en 1982, después de promulgada la Constitución de 1978 y tras ser constituidas las 17 CCAA, las competencias de salud pública, entre las que se encontraba la gestión del PNPS, fueron transferidas a nivel autonómico ${ }^{24}$.

A partir de 1983, el Real Patronato no tuvo posibilidad de continuar apoyando de manera sistemática el desarrollo del PNPS y el citado Consejo se disolvió. No obstante, el programa de detección neonatal de alteraciones metabólicas siguió expandiéndose, principalmente mediante el impulso y la tenacidad de quienes ya estaban involucrados en la práctica de la detección neonatal, y se pusieron en marcha nuevos centros.

\section{NACIMIENTO Y EVOLUCIÓN DE LOS PROGRAMAS AUTONÓMICOS}

Tras la transferencia de competencias del PNPS a las CCAA en 1982, comenzaron a surgir distintos programas de Detección 
Precoz Neonatal a nivel regional y se siguieron creando nuevos centros de cribado.

Desde aquel año hasta la actualidad, pueden distinguirse varias etapas en la evolución de los PCN españoles, que serán analizadas en posteriores revisiones:

- De 1982 a 2000: nacimiento de nuevos PCN hasta que cada autonomía implantara el suyo propio y evolución de todos los PCN clásicos o básicos hasta la irrupción de la tecnología que daría lugar a los PCN ampliados.

- De 2001 a 2015: evolución de los PCN autonómicos e implementación progresiva y heterogénea de los PCN ampliados gracias a la espectrometría de masas en tándem (MS/MS), que revolucionó el cribado neonatal de metabolopatías.

- Desde 2015: adaptación de los PCN autonómicos a la nueva normativa estatal de finales de 2014 que actualiza la cartera de servicios comunes del Sistema Nacional de Salud, regulando en parte los PCN autonómicos y estableciendo la detección precoz de, al menos, 7 enfermedades endocrino-metabólicas.

\section{BIBLIOGRAFÍA}

1. Ministerio de Sanidad, Servicios Sociales e Igualdad. Orden SSI/2065/2014, de 31 de octubre, por la que se modifican los anexos I, II y III del Real Decreto 1030/2006, de 15 de septiembre, por el que se establece la cartera de servicios comunes del Sistema Nacional de Salud y el procedimiento para su actualización. España: Boletín Oficial del Estado número 269; 6 de noviembre de 2014: 91369-91382.

2. Bearn AG, Miller ED. Archibald Garrod and the development of the concept of inborn errors of metabolism. Bull Hist Med 1979; 53 : 315-328.

3. Dronamraju K. Profiles in genetics: Archibald E. Garrod (1857-1936). Am J Hum Genet 1992; 51: 216-219.

4. SCRIVER CR. Garrod's croonian lectures (1908) and the charter Inborn errors of metabolism: albinism, alkaptonuria, cystinuria, and pentosuria at age 100 in 2008. J Inherit Metab Dis 2008; 31: 580-598.

5. GonZÁlez-Lamuño D, García-Fuentes M. Enfermedades de base genética. An Sist Sanit Navar 2008; 31 (Supl. 2): 105-126.
6. Sanjurjo P, Baldellou A, Aldámiz-Echeverría K, Montejo M, GARCía-JimÉnEz MC. Los errores congénitos del metabolismo como enfermedades raras con un planteamiento global específico. An Sist Sanit Navar 2008; 31 (Supl. 2): 55-73.

7. GonZÁlez-Lamuño D, García-Fuentes M. Enfermedades raras en pediatría. An Sist Sanit Navar 2008; 31 (Supl. 2): 21-29.

8. Gaite L, García-Fuentes M, González-Lamuño D, Álvarez JL. Necesidades en las enfermedades raras durante la edad pediátrica. An Sist Sanit Navar 2008; 31 (Supl. 2): 165-175.

9. Artuch R, Moreno J, Puig RM, Quintana M, MonTERo R, ORMAZÁBAL A et al. El laboratorio en el diagnóstico de las enfermedades raras. An Sist Sanit Navar 2008; 31 (Supl. 2): 91-103.

10. SÁnchez-Valverde F, García-Fuentes M. Enfermedades raras: el reto de la medicina en el siglo XXI. An Sist Sanit Navar 2008; 31 (Supl. 2): 5-8.

11. Posada M, Martín-Arribas C, Ramírez A, VillaverDE A, ABAItuA I. Enfermedades raras. Concepto, epidemiología y situación actual en España. An Sist Sanit Navar 2008; 31 (Supl. 2): 9-20.

12. Morabia A, Zhang FF. History of medical screening: from concepts to action. Postgrad Med J 2004; 80: 463-469.

13. Pinzón A. ¿Screening o tamizaje? Acta Médica Colomb 2012; 37: 42.

14. Delgado-Rodríguez M. Aspectos generales del cribado. En: Cerdá-Mota T, Ascunce-Elizaga N, García-García A, editores. Implantación y evaluación de programas poblacionales de cribado. España: Sociedad Española de Epidemiología, 2006: 9-28.

15. ViÑES JJ. La efectividad de la detección precoz de las enfermedades. An Sist Sanit Navar 2007; 30: 11-27.

16. Ascunce-Elizaga N. Cribado: para qué y cómo. An Sist Sanit Navar 2015; 38: 5-8.

17. Dulín-Iñiguez E, Espada M, Eguileor-Gurtubai I. Programas de cribado neonatal. An Pediatría Contin 2006; 4: 61-65.

18. GARRIDO-CuENCA NM. A vueltas sobre la prueba del talón en recién nacidos. Reflexiones a la luz de la Ley General de Salud Pública: ¿Hacia un Programa Nacional de Cribado Neonatal Ampliado? Rev Clínica Med Fam 2012; 5: 1-2.

19. Wilson JMG, Jungner G. Principles and practice of screening for disease. Geneva: World Health Organization, 1968.

20. CerdÁ-Mota T. Programas de cribado: características y condiciones para su puesta en marcha. Medicine 2003; 8: 6312-6317.

21. PAmpols-Ros T. Más no es igual que mejor. Desafíos científicos, éticos y sociales que habrá que afrontar en el cribado neonatal en la era 
de la medicina genómica. En: Trigésimo aniversario del Programa de Cribado Neonatal de la CAPV, 2012: 82-99.Disponible en:http:// www.osakidetza.euskadi.eus/contenidos/informacion/programa_cribado_neonatal/es_ cribado/adjuntos/XXX\%20Aniversario\%20 del\%20Programa\%20de\%20Cibado\%20Neonatal\%20de\%20la\%20CAPV.\%20Libro\%20 de\%20resumenes.pdf

22. Jefatura del Estado. Ley 14/2007, de 3 de julio, de investigación biomédica. España: Boletín Oficial del Estado número 159; 4 de julio de 2007: 28826-28848.

23. Calderón López MG, Jiménez Parrilla F, Losada Martínez A. Screening neonatal. En: Protocolos de Neonatologia. Asociación Española de Pediatría, 2008: 422-433. Disponible en: http://www.aeped.es/sites/default/files/documentos/44.pdf

24. Real Patronato de Prevención y de Atención a Personas con Minusvalía. Prevención de alteraciones metabólicas congénitas en España. Segunda ed. Madrid: 1998.

25. Alonso-Fernández JR, Colón C. The contributions of Louis I Woolf to the treatment, early diagnosis and understanding of phenylketonuria. J Med Screen 2009; 16: 205-211.

26. Alonso-Fernández JR, Colón-Mejeras C. Aportaciones de Louis I. Woolf al tratamiento y diagnóstico precoz de la fenilcetonuria y otros Errores Congénitos del Metabolismo. Los comienzos de la Tría Neonatal en España, con referencia al Programa de Galicia. 2015. Disponible en: https://doi.org/10.13140/ RG.2.1.5044.104.

27. Woolf LI, Vulliamy DG. Phenylketonuria with a study of the effect upon it of glutamic acid. Arch Dis Child 1951; 26: 487-494.

28. Bickel H, GerRard J, Hickmans EM. The influence of phenylalanine intake on phenylketonuria. Lancet $1953 ; 265: 812-813$.

29. BiCKEL H. The effects of a phenylalanine-free and phenylalanine-poor diet in phenylpyruvic oligophrenis. Exp Med Surg 1954; 12: 114-117.

30. Bickel H, Gerrard J, Hickmans EM. The influence of phenylalanine intake on the chemistry and behaviour of a phenylketonuria child. Acta Pædiatr 1954; 43: 64-77.

31. Woolf LI, Griffiths R, Moncrieff A. Treatment of phenylketonuria with a diet low in phenylalanine. Br Med J 1955; 1: 57-64.

32. GiBbs NK, Woolf LI. Tests for phenylketonuria: results of a one-year programme for its detection in infancy and among mental defectives. Br Med J 1959; 2: 532-535.

33. Berry HK, Sutherland B, Guest GM, Warkany J. Simple method for detection of phen- ylketonuria. J Am Med Assoc 1958; 167 : 2189-2190.

34. Centerwall WR, Chinnock RF, Pusavat A. Phenylketonuria: screening programs and testing methods. Am J Public Health Nations Health 1960; 50: 1667-1677.

35. Woolf LI. Mass screening of the newborn for metabolic disease. Arch Dis Child 1968; 43: 137-140.

36. Dussault JH. The anecdotal history of screening for congenital hypothyroidism. J Clin Endocrinol Metab 1999; 84: 4332-4334.

37. Ministerio de Hacienda. Real Decreto 1762/1977, de 10 de junio, sobre distribución de los rendimientos de la tasa sobre el juego para el ejercicio económico de 1977. España: Boletín Oficial del Estado número 168; 15 de julio de 1977: 15906.

38. Dulín-Íñiguez E, Cortés-Castell E, Chamorro-Ureña F, Eguileor-Gurtubai I, Espada-Sáez-Torre M, PÁMpols-Ros $\mathrm{T}$ et al. Actividad de los centros de detección precoz neonatal de errores congénitos del metabolismo en España. Evaluación sanitaria (1996-1999). Boletín del Real Patronato sobre Discapacidad 2001; 49: 9-23.

39. Dirección General de Salud Pública del Ministerio de Sanidad y Consumo. Cribado de enfermedades metabólicas. Programa de diagnóstico neonatal precoz de metabolopatías. Rev Pediatr Aten Primaria 2004; 6: 679-689.

40. Bernal J, OBREgón MJ. Gabriella Morreale (1930). SEBBM divulgación Galería de retratos Women in Biochemistry [Internet]. Mayo 2012 [consultado 10-10-2016]. Disponible en: http://www.sebbm.es/web/es/divulgacion/ mujeres-ciencia/retratos/371-mayo-2012-gabriela-morreale

41. SÁnchez de Mora A. Cuando la bondad se hace ciencia: Gabriella Morreale. 8 octubre 2014 [consultado 10-10-2016]. En: El quilombo del periodismo científico (blog) [Internet]. Disponible en: https://fundamentosdelperiodismocientifico.wordpress.com/2014/10/08/ gabriela-morreale-de-castro/

42. López-Rodríguez MJ, SÁnchez-Méndez JI, SÁnchezMartínez MC, Calderay-Domínguez M. Suplementos en embarazadas: controversias, evidencias y recomendaciones. Inf Ter Sist Nac Salud 2010; 34: 117-128.

43. Ministerio de Sanidad y Seguridad Social. Real Decreto 2176/1978, de 25 de agosto, por el que se encomienda al Ministerio de Sanidad y Seguridad Social la realización y gestión del Plan Nacional de Prevención de la Subnormalidad. España: Boletín Oficial del Estado número 222; 16 de septiembre de 1978: 21696-21697. 